\title{
NOTES ON A NEW METHOD FOR THE DETERMI- NATION OF THE MAGNETIC FLUX DENSITY AND PERMEABILITY*
}

\author{
$\mathrm{By}$ \\ August Hund
}

(Assistant-Professor in the Department of Physics and Electrical Engineering, University of Southern California, Los Angeles, California)

The following is an outline of a new method for determining the flux density, i. e., number of lines of magnetic induction per unit cross section, up to any desired frequency. The same arrangement may also be conveniently used for obtaining the magnetic permeability and for investigating the total loss of a coil containing a ferro-magnetic core, or the core and copper losses separately.

\section{Principle and Theory of the Method-Explanation of the Arrangement}

The suggested arrangement is shown in Figure 1 and is based on the application of a differential system, which has been recently described by the author. ${ }^{1}$ One differential branch contains the test sample which has a definite coefficient of selfinduction, $L_{x}$, for a particular current at a fixed frequency. The test sample is investigated by means of balancing its effect against a standard variable self induction, $L_{s}$, (variometer, air-core coils) in series with a non-inductive resistance $r$.

The performance of such a differential system is briefly as follows: When the currents in the two branches of the system are equal in effective value and in phase, their inductive effects on the secondary coil of the differential transformer will exactly neutralize each other, and no voltage will be induced in the coil. This is based on the assumption that the two primary coils, $P_{1}$ and $P_{2}$, are symmetrically placed with reference to the secondary coil, $S$, and have exactly the same number of turns which are wound in opposite directions. Any kind of alternating cur-

\footnotetext{
${ }_{1}^{*}$ Received by the Editor, October 1, 1916.

${ }^{1}$ A. Hund, "Electrical World," May' 22, 1915; reprinted in "London Electrician," August 27, 1915.
} 
rent detector connected across the terminals of the secondary coil will then give a no-current indication when the currents in $P_{1}$ and $P_{2}$ are equal and in phase.

A no-current adjustment is established when the coefficient of self-induction of the variometer is equal to the effective coefficient of self induction of the test coil and when the effective resistance of the test coil is exactly balanced by the resistance

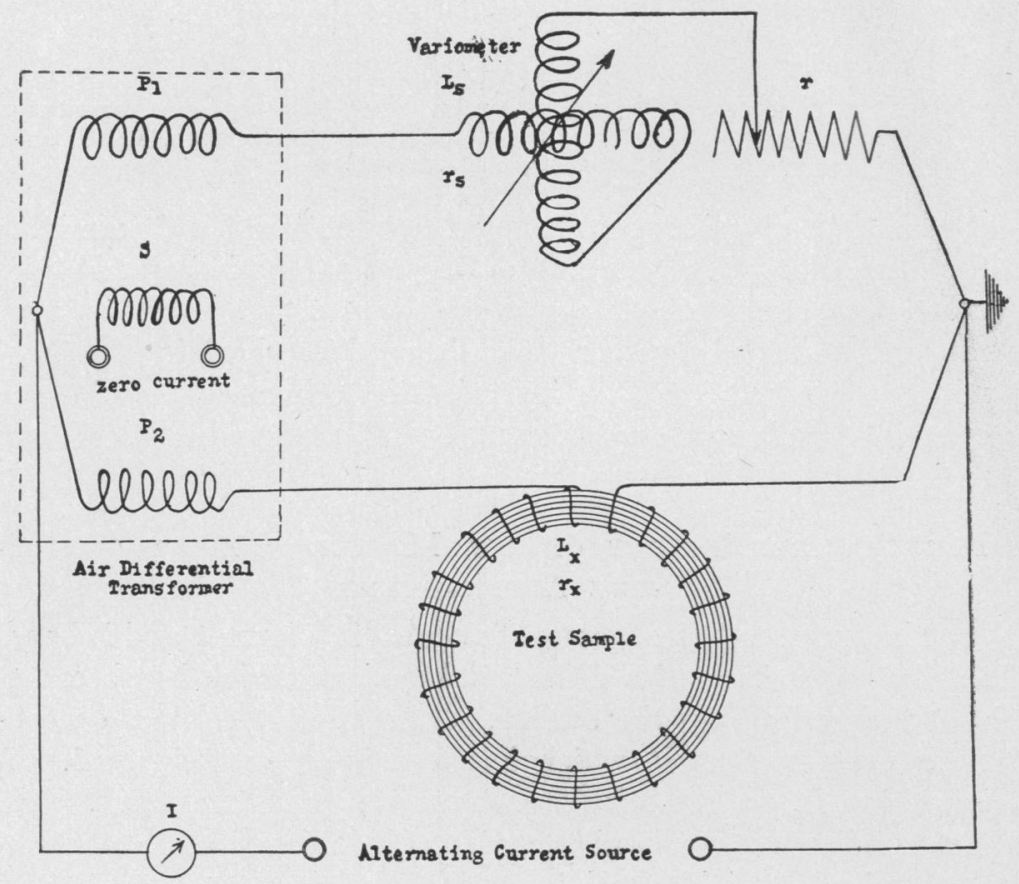

Figure 1

of the variometer and the series resistance $r$. An absolute disappearance of the differential field, however, can generally not be obtained since the wave form in the one branch is somewhat distorted due to the presence of the ferro-magnetic substance. In most practical cases, however, the minimum of the differential field can be very readily and accurately detected. For very precise measurements, it is advantageous to insert a condenser in series with the indicator in the secondary circuit of the differential transformer and tune this circuit to resonance with the required frequency. (This is especially recommended at higher 
frequencies since a tuning is then readily obtainable.) For frequencies up to about 2,000 cycles, a Wien vibration galvanometer may be used as a current indicator, and the condenser may accordingly be dispensed with.

Derivation of the Expression of the Maximum Flux

\section{Density}

When the ferro-magnetic core of a coil is exposed to an alternating flux of maximum value, $\Phi_{\max }$, the total change in the lines of induction, which go thru the cross sectional area of the iron core, during one half wave, is from zero to $\Phi_{\max }$ and back to zero, i. e., a total change of $2 \Phi_{\max }$. When $f$ denotes the number of cycles per second and $T$ the corresponding, period, the average rate of change is $\frac{2 \Phi_{\max }}{\frac{T}{2}}$. Hence the average in-

duced voltage per each turn of the coil is

$$
E_{a v}=4 f \Phi_{\max } 10^{-8} \text { volts }
$$

and the effective value for $N$ turns is equal to

$$
E=4 F f N \Phi_{\max } 10^{-8} \text { volts }
$$

where $F$ denotes the form factor of the voltage wave, i. e., the ratio of effective value to average value. In case the flux traversing the core follows a sine law, the instantaneous value of it at any time, $t$, is defined as

$$
\Phi_{t}=\Phi_{\text {max }} \sin (2 \pi f t)
$$

The form factor $F$ as determined according to the above definition is

$$
F=\frac{\sqrt{\frac{2}{T} \int_{o}^{\frac{\mathrm{T}}{2}} e^{2} d t}}{\frac{2}{T} \int_{0}^{\frac{T}{2}} e d t}=\frac{\frac{E_{\max }}{\sqrt{2}}}{\frac{2 E_{\max }}{\pi}}=1.111
$$

and equation (2) becomes

$$
E=4.44 f N \Phi_{\max } 10^{-8} \text { volts }
$$

When this expression is applied to the arrangement under discussion and the maximum flux density, $B_{\max }$, is introduced, we find the expression for the induced E. M. F. of the test sample as

$$
E=4.44 f N S B_{\max } 10^{-8} \text { volts }
$$

in which relation $S$ stands for the cross sectional area of the 
iron core. Now let the two inductances, $L_{x}$ and $L_{s}$, be adjusted to the same value; i. e., first, adjustment of phase, and the resistance, $r$, regulated until the indicator of the differential transformer shows no effect whatever, and second, adjustment of amplitude. Then we may write ${ }^{2}$

$$
4.44 f N S B_{\max } 10^{-8}=2 \pi f L_{s} \frac{I}{2}
$$

which leads to

$$
B_{\max }=0.7075 \frac{L_{\mathrm{s}(\mathrm{henrys})}}{N S_{(\mathrm{cm.}))^{2}}} I_{\text {(amps.) }} 10^{8} \quad \begin{gathered}
\text { lines of induction } \\
\text { per square centimeter }
\end{gathered}
$$

the expression for the maximum flux density in terms of $L_{s}$ as read on the variometer; $I$, as measured by the ammeter in the main branch of the differential arrangement; $N$, the number of turns of the test sample; and, $S$, the cross sectional area of the iron core.

Determination of the Resultant Field Intensity $H_{\max }$ and Magnetic Permeability $\mu$

One way of exploring the magnetic properties of a ferromagnetic substance by means of this method is to use a circular ring on which is uniformly wound a coil of wire of comparatively low resistance. Then the maximum magnetizing force, $H_{\max }$, is defined by the formula ${ }^{3}$

$$
H_{\max }=\frac{4 \pi}{10} \sqrt{2} \frac{N I_{m \text { (amps) }}}{l_{\mathrm{cm}}} \begin{gathered}
\text { gilberts per } \\
\text { centimeter }
\end{gathered}
$$

where $I_{m}$ is the magnetizing component of the effective current traversing the coil of the sample, $N$ the number of turns in the coil, and $l$ the mean length of the magnetic path in centimeters. It is to be borne in mind that this equation holds only approximately when the diameter of the ring is large as compared with the diameter of the cross section. Suppose that in Figure 2 $L_{x}$ and $r_{x}$ denote the effective coefficient of self induction and apparent resistance of the sample and that they are exactly balanced by $L_{s}$ and $\left(r_{s}+r\right)$; that is, the self induction of the variometer, the resistance of it, and the additional balance resistance $r$. The vector diagram of Figure 3 then shows the voltage relations in the test coil and objects used for comparison. In this diagram $V$ denotes the effective terminal pressure of the test sample and $\phi$ the phase difference between $V$ and the branch current $I / 2$. If the ohmic resistance of the sample, for direct current, is denoted by $r_{x}{ }^{\prime}$ and if $\Delta r_{f}$ represents the increase in

\footnotetext{
${ }^{2}$ This is only approximately true since the resistance adjustment changes the phase also to a certain extent.

${ }^{3} 1$ gilbert $=0.79578$ ampere-turn.
} 
resistance due to skin effect at the frequency $f$, and since the increase of resistance due to hysteresis and eddy current losses is given by the term

$$
\frac{\text { total iron losses }}{\left(\frac{1}{2}\right)^{2}}
$$

the apparent resistance, $r_{x}$, of the sample is defined by

$$
\begin{aligned}
r_{x} & =r_{x}{ }^{\prime}+\Delta r_{f}+\frac{W_{c}}{\left(\frac{I}{2}\right)^{2}} \\
& =r_{s}+r
\end{aligned}
$$

where $W_{c}$ stands for the total core loss in the ferro-magnetic substance. To equate $r_{x}$ to the quantity $\left(r_{s}+r\right)$ is correct if

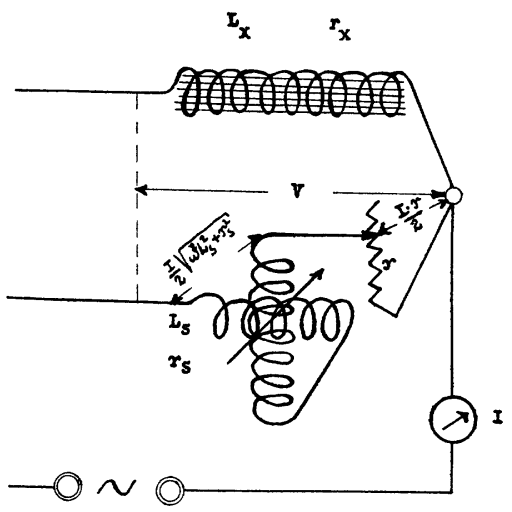

Figure 2

we assume that the wire used for the variometer shows no appreciable skin effect, so that its direct current resistance $r^{\prime}=r$ is equal to the alternating current resistance $r^{\prime \prime}$. Within the range of the very high (radio) frequencies such an assumption can not be made, even if ideal twisted wires or ribbons are used, such as described by the author in a previous publication, ${ }^{2}$ and the high frequency resistance of the variometer is to be determined by the well known methods if a calibration curve is not available. The magnetizing current $I_{m}$ which is to be introduced in the equation (7) can be expressed in terms of the effective

${ }^{4}$ A. Hund, "Arbeiten aus dem Elektrotech. Institut der Technischen Hochschule, Karlsruhe," Volume III. 
current thru the test sample. For the balanced differential system we obtain

$$
I_{m}=\frac{I}{2} \sin \phi
$$

This equation is based on the assumption that the resistance of the windings of the sample is small as compared with the inductive reactance, a requirement which is easily fulfilled, espe-

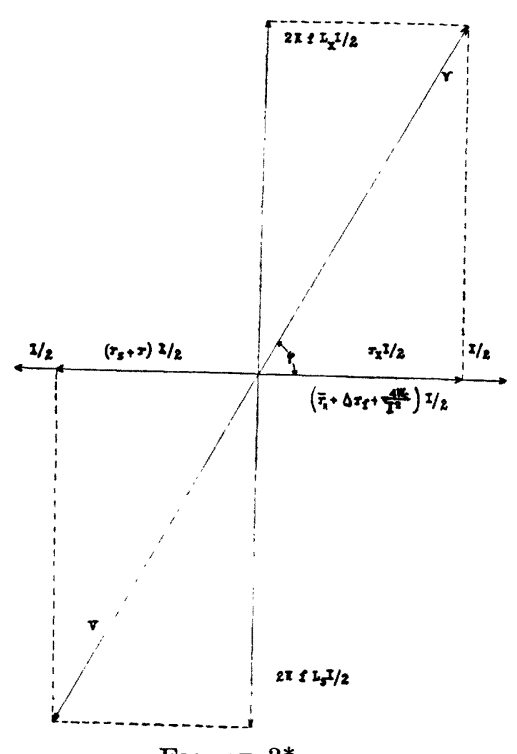

Figure $3^{*}$

cially at higher frequencies where the wattless components usually become very pronounced. The proof of this approximation may be readily seen from the following consideration:

The total iron loss of the test sample produces an increase in current and decrease in phase displacement, $\phi$, between impressed voltage and current under the conditions of constant terminal voltage. That is, the iron acts like a secondary circuit which is coupled to the coil of the sample. This fact is shown in Figure 4 where $A B C$ denotes the impedance triangle for the sample coil without iron, for which

$\overline{A B}=2 \pi f L_{x}$, the inductive reactance

$\overline{B C}=r_{x}{ }^{\prime \prime}=r_{x}{ }^{\prime}+\Delta r_{f}$, the effective ohmic resistance at the frequency $f$ and

${ }^{*}$ Wherever an $r$ with a dash $(-)$ over it appears in a figure, it corresponds to $r^{\prime}$ in the text; similarly an $r$ with a cycle mark $(\sim)$ over it corresponds to $r^{\prime \prime}$ in the text. 
$\overline{A C}^{\prime}=Z_{x}$

$=\sqrt{\left(2 \pi f L_{x}\right)^{2}+r_{x}{ }^{\prime \prime 2}}$, the resistance operator of the coil.

The impedance triangle goes over into the triangle $A^{\prime} B C^{\prime}$ for the same terminal voltage when the iron core is added, which represents the true conditions of the sample. In this case we have

$\overline{A^{\prime} B}=2 \pi f\left(L_{x}-\Delta L_{x}\right)$, the inductive reactance $\overline{B C^{\prime}}=r_{x}=r_{x}{ }^{\prime \prime}+\frac{W_{c}}{\left(\frac{I}{2}\right)^{2}}$, the apparent ohmic resistance

$\overline{A^{\prime} C^{\prime}}=Z_{x}^{\prime}$

$$
=\sqrt{\left[2 \pi f\left(L_{x}-\Delta L_{x}\right)\right]^{2}+\left[r_{x}^{\prime \prime}+\frac{W_{c}}{\left(\frac{I}{2}\right)^{2}}\right]^{2}}
$$

for a

certain

current of definite frequency.

the resistance operator of the coil

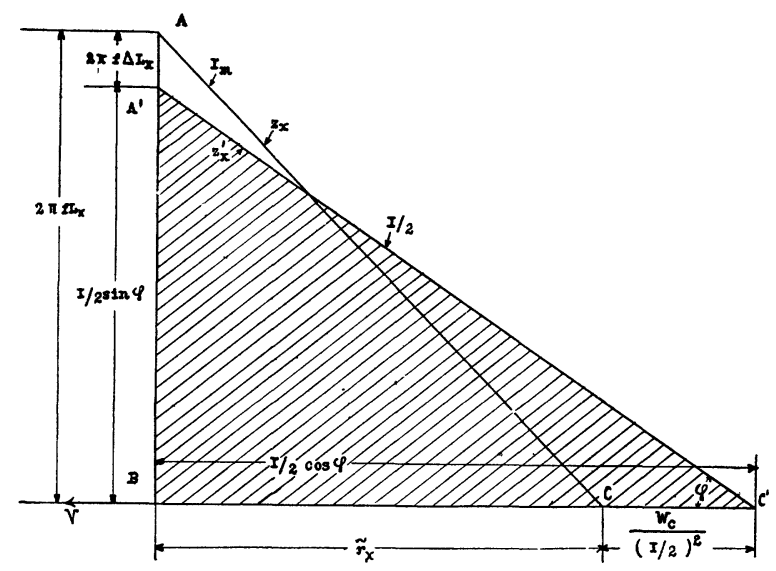

Figure 4

The angle $A^{\prime} C^{\prime} B$ represents the actual displacement of phase between impressed voltage and current traversing the coil. A little further analytical reasoning will show that the decrement in self inductance due to the presence of the ferro-magnetic substance is given by the expression

$$
\Delta L_{x}=\frac{(2 \pi f M)^{2} L_{2}}{r_{2}^{2}+\left(2 \pi f L_{2}\right)^{2}}
$$


and the increment in resistance due to the presence of the ferromagnetic substance by

$$
\frac{W_{c}}{\left(\frac{I}{2}\right)^{2}}=\frac{(2 \pi f M)^{2} r_{2}}{r_{2}{ }^{2}+\left(2 \pi f L_{2}\right)^{2}}
$$

in which case we imagine the iron core to be substituted by a secondary circuit of self inductance $L_{2}$ and ohmic resistance $r_{2}$. $\quad M$ denotes then the mutual inductance between the coil of the test sample and the fictitious turns of the secondary. We know from the theory of alternating currents that the current triangle is similar to the impedance triangle; which, when applied to our case, means that Figure 4 simultaneously represents the current relations. The scale, of course, would have to be properly selected. Thus the shaded triangle, the case where a ferro-magnetic substance is present, is as follows:

$\overline{A^{\prime} B}=\frac{I}{2} \sin \phi$, the wattless component

$\overline{B C^{\prime}}=\frac{I}{2} \cos \phi$, the watt component

$$
\begin{aligned}
& \text { of the } \\
& \text { current } \\
& \text { in the } \\
& \text { coil }
\end{aligned}
$$

$$
\begin{aligned}
& \overline{A^{\prime} C^{\prime}}=\frac{I}{2}, \text { the actual current passing thru the coil of } \\
& \text { the ammeter reading in the main branch } \\
& \text { of the differential system. }
\end{aligned}
$$

The triangle $A B C$ represents the vector diagram for the currents when no iron core is present and the hypothenuse denotes the magnetizing current $I_{m}$ which is utilized in equation (7) for the evaluation of the magnetizing force $H_{\max }$.

Returning to equation (10) we learn that $\Delta L_{x}$ is only a very small quantity when the term, $r_{2}{ }^{2}$, in the fictitious secondary circuit is large as compared with the term $\left(2 \pi L_{2}\right)^{2}$. This is true in our case to a fair degree of approximation when we assume that the eddy currents are induced in a well subdivided iron core, such as is usually employed in alternating current practice at higher frequencies. With this assumption, the point $A$ and $A^{\prime}$ may be thought of as coinciding, which leads to a current diagram such as is shown in Figure 5. The base B $C^{\prime}$ represents, to a certain scale the total power input for the test sample containing a ferro-magnetic core since $C C^{\prime}$ denotes the input due to hysteresis and eddy current loss, and $B C$ the input due to copper loss in the turns of the sample. If the resistance of the sample is kept small in comparison with the inductive reactance, as indicated in Figure 5, the magnetizing current, 
$I_{m}$, is only a little smaller than the wattless component of the true and measurable coil current. This means that for a thinly laminated iron core and using the so-called ideally twisted wire ${ }^{5}$ in the test coil of rather low resistance and comparatively high self inductance, the sine component of the branch current passing

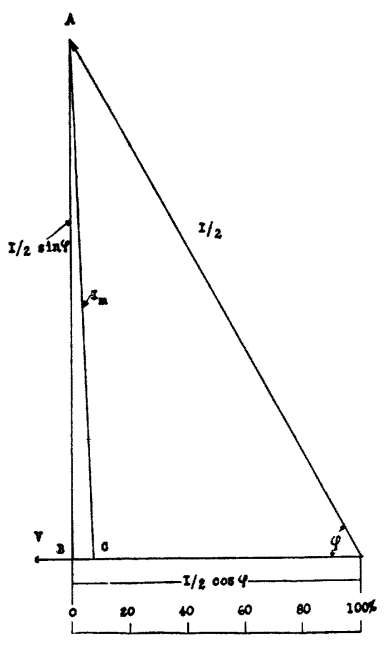

Figure 5

thru the sample denotes to a fair degree of approximation the magnetizing current $I_{m}$. Returning to equation (9) we find there the magnetizing force, $H_{\max }$, and the magnetic permeability, $\mu$, by the following procedure:

$$
\begin{aligned}
\sin \phi & =\frac{2 \pi f L_{x}}{\sqrt{\overline{r_{x}^{2}+\left(2 \pi f L_{x}\right)^{2}}}} \\
& =\frac{2 \pi f L_{s}}{\sqrt{\left(r_{s}+r\right)^{2}+\left(2 \pi f L_{s}\right)^{2}}}
\end{aligned}
$$

then the magnetizing current becomes

$$
I_{m}=\frac{I}{2} \cdot \frac{2 \pi f L_{s}}{\sqrt{\left(r_{s}+r\right)^{2}+\left(2 \pi f L_{s}\right)^{2}}}
$$

and according to equation (7) the maximum magnetizing force is defined as

$$
H_{\text {max }}=5.57 \frac{N f L_{s(h e n r y)} \cdot I_{(a m p s)}}{l_{(c m)} \sqrt{\left(r_{s(o h m)}+r_{(o h m)}\right)^{2}+2 \pi f L_{s(h e n r y))^{2}}}} \text { centimeter }
$$

5 "Litzendraht." 
and the magnetic permeability is given by the relation

$$
\begin{aligned}
\mu & =\frac{B_{\max }}{H_{\max }} \\
& =1.27 \frac{l_{(\mathrm{cm})} \sqrt{\left(r_{s(o h m)}+r_{(o h m)}\right)^{2}+\left(2 \pi f L_{s(h e n r y)}\right)^{2}}}{N^{2} \cdot S_{(\mathrm{cm})} f} 10^{7}
\end{aligned}
$$

When the wave length, $\lambda$, is introduced, as is often customary within the range of radio frequencies, the maximum magnetizing force and magnetic permeability may be determined by the following relations ${ }^{6}$ :

$$
H_{\text {max }}=16.71 \frac{N \cdot L_{s(h e n r y)} I_{(a m p s)}}{\lambda_{(m)} l_{(c m)} \sqrt{\left(r_{s(o h m)}+r_{(o h m)}\right)^{2}+\left(\frac{6 \pi \cdot 10^{8} L_{s(h e n r y)}}{\lambda_{(m)}}\right)^{2}}} \times 10^{8}
$$

gilberts per centimeter

and

$$
\mu=42.33 \frac{\lambda_{(m)} l_{(\mathrm{cm})} \sqrt{\left(r_{s(o h m)}+r_{(o h m)}\right)^{2}+\left(\frac{6 \pi \cdot 10^{8} L_{s(h e n r y)}}{\lambda_{(m)}}\right)^{2}}}{N^{2} S_{(c m)}} \times 10^{-3}
$$

We therefore see that for a standard test ring of given cross sectional area, length of magnetic path and number of turns, the maximum flux density, $B_{m a x}$, the maximum resultant field intensity, $H_{\max }$, and the permeability, $\mu$, can be calculated from observed data by means of the three following formulae:

$$
\begin{array}{cc}
B_{\max }=k_{1} L_{s(h e n r y)} I_{(a m p s)} & \begin{array}{l}
\text { lines of induction per } \\
\text { square centimeter }
\end{array} \\
H_{\max }=k_{2} \frac{f L_{s(\text { henry })} I_{(a m p s)}}{Z_{(\text {ohms })}} & \text { gilberts per centimeter } \\
& \mu=k_{3} \frac{Z_{(o h m s)}}{f}
\end{array}
$$

where $z$ is equivalent to the resistance operator of the test sample and the constants $k_{1}, k_{2}$, and $k_{3}$ of a definite dimensioned sample are given by the relations:

$$
\begin{aligned}
k_{1} & =\frac{0.7075 \times 10^{8}}{N \cdot S_{(\mathrm{cm})^{2}}} \\
k_{2} & =5.57 \frac{\mathrm{N}}{l_{(\mathrm{cm})}} \\
k_{3} & =1.27 \frac{l_{(\mathrm{cm})}}{N^{2} S_{(\mathrm{cm})^{2}}} 10^{7}
\end{aligned}
$$

${ }^{6}$ In above formulas, both meters and centimeters are purposely employed, since the wave meters are usually calibrated in meters and the length of the magnetic path is generally measured in centimeters. To express also the self induction in the C. G. S. system was not done since most of the commercial variometers are calibrated in practical units. 
A few concluding remarks on the true magnetizing current, $I_{m}$, such as utilized for the calculation of the magnetizing force, are given here, before the determination of the core losses is discussed. Such considerations may not be entirely new, altho it seems worth while to add a more detailed analysis in direct application to the differential system, and to derive other expressions with which to calculate the magnetizing force and the permeability.

One should first clearly distinguish between exciting current and magnetizing current, since in ordinary engineering discussions both expressions are often used interchangeably to denote the same quantity, namely, the no-load current of the transformer and the feeding current of a choke coil, respectively. Exciting current of the test sample is the total flow of electricity that passes thru the coil. It is denoted by $I / 2$ for the balanced differential system, and is obtained from the ammeter reading of the main branch of the arrangement. This current includes that consumed as Joulean heat losses in the windings of the sample, and also for supplying the losses due to hysteresis and eddy currents. The exciting current may therefore be split up into an energy component, determined by the total loss which is in the vectorial direction of the terminal pressure, $V$, and into the magnetizing component, $I_{m}$, which is in the vectorial direction of the magnetic induction, $B$, i. e., wattless. Now, if the flux density of the ferro-magnetic medium is within the range of the straight part of the magnetization curve, the magnetizing current, $I_{m}$, will vary according to a sine law when the flux is sinusoidal. But in case of higher saturations for which the flux density rises beyond the straight part of the saturation curve (for instance, beyond the knee), the magnetizing current becomes distorted. Consequently, the exciting current which a sinusoidal impressed E. M. F. will establish in the turns of our test sample no longer varies according to a sine law. This can be seen from Figure 6 where the instantaneous current values, $i / 2$, of the test coil, such as have been obtained from the hysteresis loop, are plotted against the time. It is to be noted that the ohmic drop in the winding is considered as being small enough to be neglected in this representation, so that it is possible to assume that the induced E. M. F. is at all times equal and opposite to the impressed terminal voltage, $V$, of the sample. The figure clearly shows that the exciting current, $i / 2$, required to produce a sinusoidal flux density wave is unsymmetrical with respect to its maximum ordinate. The maximum flux density occurs at 


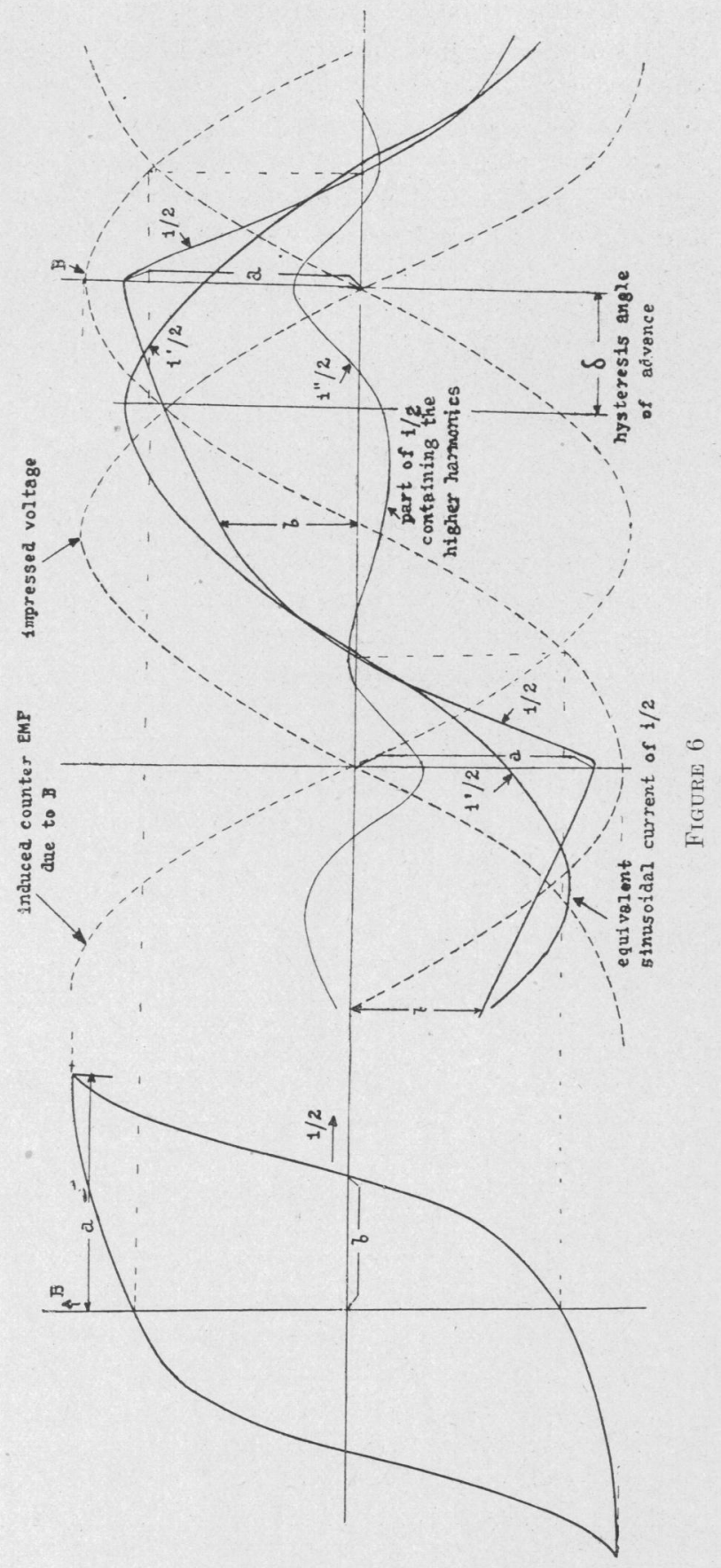


the same time that the exciting current reaches its maximum; that is to say, this current is 90 time-degrees ahead of the induced E. M. F. and generally about 90 time-degrees behind the impressed voltage, whereas the intersection with the zero line indicates a considerable lead with respect to the zero value of the flux density. The wave of the exciting current of commercial frequency up to the highest frequencies, such as employed in radio telegraphy and telephony, is usually distorted by the presence of higher harmonics of a very pronounced triple harmonic. As is indicated above, the distortion is chiefly due to the magnetizing current, $I_{m}$, and is caused on account of the curved part of the $B-H$ curve. With transformers, this distortion is greatly diminished by the load current which, when large enough, makes insignificant the well defined distorting component of the exciting current. In our case, however, where a closed magnetic circuit is often employed, the distortion is very pronounced and an analysis of the exciting circuit is accordingly of interest. One way of studying the distorted exciting current is to split it up into those components which are in phase with the induced E. M. F. (and are often called the hysteresis and power components), and one component 90 time-degrees behind it (that is, in phase with the magnetic induction, $B$, and representing the magnetizing current). Such a resolution of the exciting current is shown in Figure 7, which represents a typical case. It is readily seen

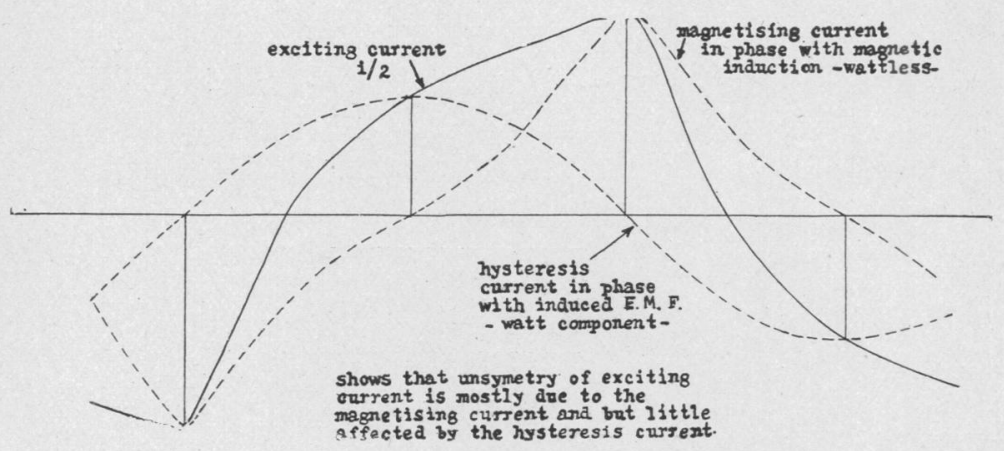

Figuke 7

that the distortion of the exciting current, such as is obtained from the hysteresis loop, is chiefly influenced by the magnetizing component as a consequence of the non-proportionality of the 
$B-H$ relation, whereas the hysteresis current wave is a good approximation a sine curve. (The reader who would like to pursue this subject in more detail will find a very interesting treatment in Dr. Steinmetz's "Alternating Current Phenomena.") In Figure 6, the exciting current is, however, resolved into a first harmonic component of the same power and effective value as that of the exciting current curve and into a component containing the higher harmonics. The latter component, which is composed of the higher harmonics, is wattless with respect to the sinusoidal applied voltage of fundamental frequency and consequently the effective watt component, $I^{\prime}{ }_{w} / 2$, of the equivalent sine wave, $i^{\prime} / 2$, denotes the total power component of the exciting current and is equivalent to $I_{h}$, the hysteresis current. The magnetizing current, $I_{m}$, is then constituted of the wattless component, $I^{\prime}{ }_{w L} / 2$, of the equivalent sine wave, $i^{\prime} / 2$, and the effective value $I^{\prime \prime} / 2$ of the curve, $i^{\prime \prime} / 2$, containing the higher harmonics, chiefly of triple frequency. This is made plainer by the vector diagram of Figure 8, which shows the construction of the effective value, $I / 2$, of the exciting current, $i / 2$; which, by assumption, is also equal to the effective value of the equivalent. sine curve. The vector, $I / 2$, makes the angle, $\delta$, with the true magnetizing current. The same is called the angle of hysteretic phase advance, and denotes the angle by which the first harmonic of the exciting current leads the sinusoidal wave of the flux density, $B$. The effective value of the measurable coil current of the test sample is therefore given by the relation

$$
\frac{I}{2}=\sqrt{\left[\frac{I_{w}^{\prime}}{2}\right]^{2}+\left[\left(\frac{I_{w L}^{\prime}}{2}\right)^{2}+\left(\frac{I^{\prime \prime}}{2}\right)^{2}\right]^{2}}
$$

and the effective value of the true magnetizing current entering the equation (7) becomes

$$
\begin{aligned}
I_{m} & =\sqrt{\left[\frac{I^{\prime}{ }_{w L}}{2}\right]^{2}+\left[\frac{I^{\prime \prime}}{2}\right]^{2}} \\
& =\sqrt{\left[\frac{I}{2}\right]^{2}-\left[\frac{I^{\prime}{ }_{w}}{2}\right]^{2}}
\end{aligned}
$$

which, translated into technical language, states that the power component demagnetizes the iron core. This can be made plainer by drawing the hysteresis component, $-I_{h}$, equal and opposite to, $I_{h}$. Then the vectors, $-I_{h}$ and $I / 2$, constitute the magnetizing current, $I_{m}$. The diagram shows furthermore that for zero hysteresis effect, the magnetizing current would be identical with 
the exciting current, $I / 2$, altho the wave would be still unsymmetrical due to the varying permeability, $\mu$.

The power, $W_{h}$, dissipated because of the hysteresis can be determined by this method, as is shown in a later paragraph. The effective current $I / 2$ is obtained from the ammeter reading of the balanced arrangement and the applied terminal voltage, $V$, is found from the relation:

$$
\begin{aligned}
V & =\frac{I}{2} \sqrt{r_{x}^{2}+\left(2 \pi f L_{x}\right)^{2}} \\
& =\frac{I}{2} \sqrt{\left[r+r_{s}\right]^{2}+\left[2 \pi f L_{s}\right]^{2}}
\end{aligned}
$$

The magnetizing current is then determined according to the procedure

$$
\begin{aligned}
W_{h} & =V \cdot \frac{I}{2} \cos (90-\delta) \\
& =V \cdot \frac{I}{2} \sin \delta \\
& =V \cdot \frac{I^{\prime}{ }_{w}}{2}
\end{aligned}
$$

introduced in equation (18)

$$
I_{m}=\sqrt{\left[\frac{I}{2}\right]^{2}-\left[\frac{W_{h}}{V}\right]^{2}}
$$

This equation, however, is based on the assumption that the ohmic drop in the test sample is negligibly small and the losses due to eddy currents are accordingly ignored. The first assumption may be readily satisfied by employing so-called "ideal"twisted wire of low resistance, while the effect due to eddy currents has to be taken into account, especially when taking readings within the range of radio frequencies. It is known that the eddy currents, like magnetic hysteresis, cause the phase of the current, to advance; the angle of which phase advance can be calculated from its sine, which is defined by the ratio of the absolute admittance of the circuit to the eddy current conductance. For well laminated iron cores, the distortion of the current wave may be kept very small, as can be demonstrated by investigating the hysteresis loops of different laminated samples by means of a Braun tube. The distortion of the hysteresis loop, due to eddy currents, is caused because they act like secondary circuits and consequently their magnetic fields counteract the main field of the coil. For this reason, in the case of a fixed magnetizing force, the total flux density is smaller when eddy currents are 
present than without it. Hysteresis loops such as were found in the splendid researches of Prof. Max Wien ${ }^{7}$ and others show this effect very plainly. Furthermore, the loops show rounded corners and are more inclined to the axis of the magnetizing force at higher frequencies, altho the area of the loop seems to change but little as the frequency increases. Another important in-

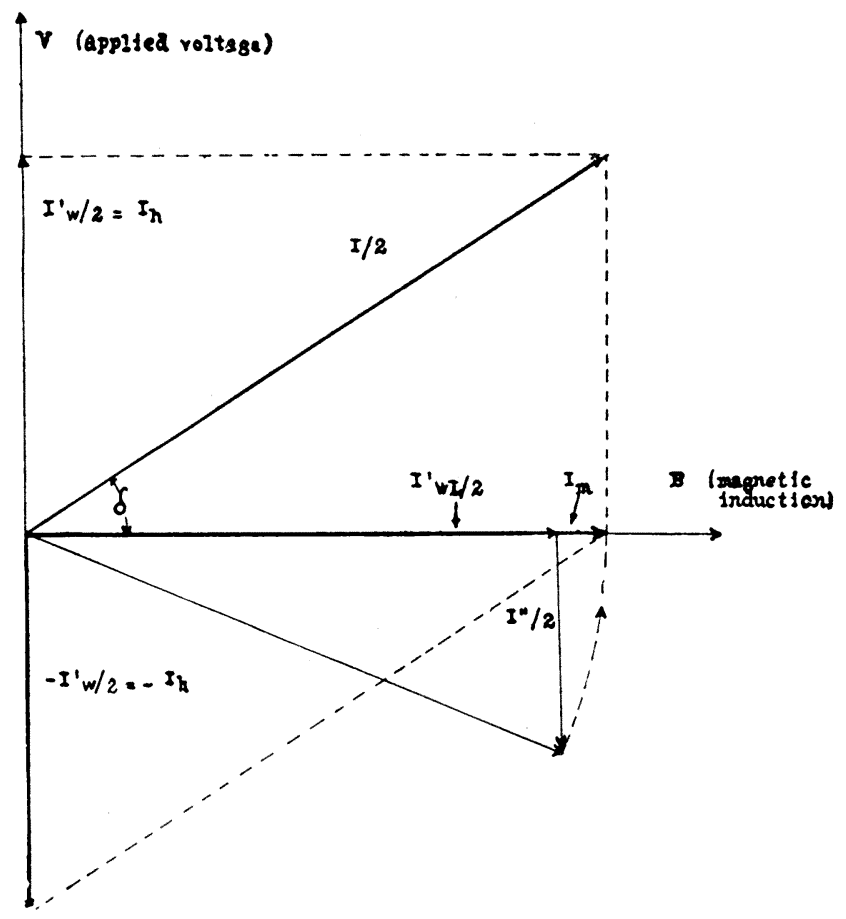

Figure 8

fluence of the eddy currents on the hysteresis loss, which is especially pronounced at higher frequencies, is that the lines of magnetic induction are not uniformly distributed over the cross section of the iron core.

Nevertheless, the measurements described in the above paragraph are not any more difficult when the effect of the eddy currents is taken into account; for the flux density wave (and along with it the eddy currents) also follow a sine law with a sinusoidal terminal voltage. The eddy currents simply increase the watt

${ }^{7}$ M. Wien, “Ann. der Physik," 1898. 
and wattless components of the equivalent sine wave of the exciting current on account of the larger exciting current. This means that the general formula of equation (21) is given by the expression

$$
I_{m}=\sqrt{\left[\frac{I}{2}\right]^{2}-\left[\frac{W_{c}}{V}\right]^{2}}
$$

and, according to a derivation given in a later paragraph, the true magnetizing current may be found from

$$
I_{m}=\sqrt{\left[\frac{I}{2}\right]^{2}-\left[\frac{\left(\frac{I}{2}\right)^{2} \Delta r_{c}}{\frac{I}{2} \sqrt{r_{x}^{2}+\left(2 \pi f L_{x}\right)^{2}}}\right]^{2}}
$$

or

$$
I_{m(a m p s)}=\frac{I_{(a m p s)}}{2} \sqrt{1-\frac{\left(\Delta r_{c(o h m)}\right)^{2}}{\left(r_{(o h m)}+r_{s(o h m)}\right)^{2}+\left(2 \pi f L_{s(h e n r y)}\right)^{2}}}
$$

which leads to the expressions

$$
H_{\text {max }}=0.8875 \frac{N \cdot I_{(a m p s)} \sqrt{1-\frac{\left(\Delta r_{c(o h m)}\right)^{2}}{\left(r_{(o h m)}+r_{s(o h m)}\right)^{2}+\left(2 \pi f L_{s(h e n r y)}\right)^{2}}}}{\begin{array}{c}
l_{(c m)} \\
\text { gilberts per centimeter }
\end{array}}
$$

and

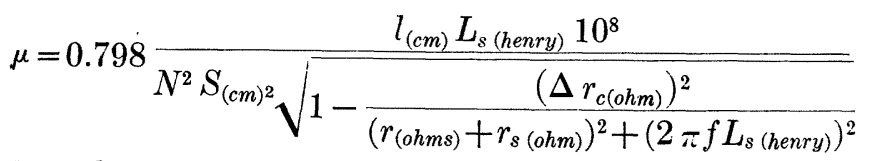

We therefore see that for a standard test ring of given cross sectional area, length of magnetic path and number of turns, the maximum resultant field intensity, $H_{m a x}$, and the magnetic permeability, $\mu$, can be calculated from observed data by means of the following formulas:

and

$$
H_{m a x}=k_{4} I_{(a m p s)} \sqrt{1-\left(\frac{\left.\Delta r_{c(o h m)}\right)}{z_{(o h m)}}\right)^{2}} \begin{aligned}
& \text { gilberts per } \\
& \text { centimeter }
\end{aligned}
$$

$$
\mu=k_{5} \frac{L_{s(\text { henry })}}{\sqrt{1-\left(\frac{\Delta r_{c(o h m)}}{z_{(o h m)}}\right)^{2}}}
$$

where $z$ represents again the resistance operator of the test sample and constants $k_{4}$ and $k_{5}$ are given by the expressions

and

$$
\begin{aligned}
& k_{4}=0.8875 \frac{N}{l_{(c m)}} \\
& k_{\overline{0}}=0.798 \times 10^{8} \frac{l_{(\mathrm{cm})}}{N^{2} S_{(\mathrm{cm})^{2}}}
\end{aligned}
$$




\section{Determination of Iron Losses}

If the magnetic field of a coil is replaced by a ferro-magnetic field, the number of lines of induction is increased, which in turn necessitates an increase in the coefficient of self induction of the coil. Since the flux does not increase as the current traversing the coil on an iron core, the coefficient of self finduction is not a constant for a certain frequency, but is a function of the current. Because the iron core is a consumer of energy, the ohmic resistance of the coil will apparently increase (equation (8)). This means that the quantity, $r_{x}$, also depends on the current. The differential arrangement therefore is a ready means to determine the iron losses. The procedure is simply this:

Measure the resistance, $r_{x}{ }^{\prime}$, of the test sample without iron by means of direct current. Adjust the resistance of the variometer combination to the same value by regulating the series resistance, $r$. Then apply the desired high frequency current of a definite frequency, $f$, to the differential system and increase $r$ in the variometer branch by the quantity, $\Delta_{r_{f}}$; that is, until an amplitude adjustment is attained. It is also advisable simultaneously to balance the phases of the two differential branches by making, $L_{s}=L_{x}$, since then the amplitude balance is more easily obtainable under such conditions. The additional resistance, $\Delta r_{f}$, then represents the increase of the ohmic resistance due to skin effect, which produces the additional loss, $\Delta r_{f}\left(\frac{I}{2}\right)^{2}$. Now insert the iron core in the coil, adjust the phase by again varying the standard self inductance (that is, until, $L_{s_{c}}=L_{x_{\mathrm{c}}}$ ), where $L_{x_{c}}$ denotes the effective coefficient of self induction of the test coil in the presence of iron for a certain wave length and a definite current value. Then add the resistance, $\Delta r_{c}$, until a complete balance is reached. The quantity, $\Delta r_{c}$, stands then for the increase of resistance due to core loss for a certain current and wave length, which leads to the following relations:

$$
\begin{aligned}
W_{c} & =\left[\frac{I}{2}\right]^{2} \Delta r_{c} \\
& =W_{h}+W_{e} \\
& =\eta \cdot f v B_{\max }^{a} 10^{-7}+\xi f^{2} d^{2} v B_{\max }^{\beta} 10^{-14}
\end{aligned}
$$

where $W_{c}, W_{e}, W_{h}$ denote: the total core loss, eddy current loss, hysteresis loss in watts, $\eta$ the hysteresis coefficient, $\xi$ the eddy current coefficient, $v$ the volume of the iron in $\mathrm{cm} .{ }^{3}$ $B_{\max }$ the maximum number of lines of induction per $\mathrm{cm} .{ }^{2}$ $d$ the thickness of the iron laminations in $\mathrm{cm}$. The ex- 
ponents $a$ and $\beta$ can be determined by observations at different flux densities. The separation of the hysteresis and the eddy current losses may be carried on in the usual way, when observations are taken for the same flux density at two different frequencies, $f_{1}$ and $f_{2}$, using the following expressions

$$
\begin{aligned}
\frac{W_{c_{1}}}{f_{1}} & =\eta v B_{\text {max }}^{a} 10^{-7}+\xi f_{1} d^{2} v B_{\max }^{\beta} 10^{-14} \\
& =K_{1} B_{\max }^{a}+K_{2} f_{1} B_{\max }^{\beta} \\
& =A+f_{1} D \\
\frac{W_{c_{2}}}{f_{2}} & =\eta v B_{\max }^{a} 10^{-7}+\xi f_{2} d^{2} v B_{\max }^{\beta} 10^{-14} \\
& =K_{1} B_{\max }^{a}+K_{2} f_{2} B_{\max }^{\beta} \\
& =A+f_{2} D
\end{aligned}
$$

and

where, $A$, denotes the hysteresis loss in watts per cycle and, $f \cdot D$, the eddy current loss in watts per cycle. Hence

and

$$
\begin{aligned}
A & =\frac{\frac{W_{c_{1}} \cdot \dot{f}_{2}}{f_{1}}-\frac{W_{c_{2}} \cdot f_{1}}{f_{2}}}{f_{2}-f_{1}} \\
& =0.333 . . \frac{W_{c_{1}} \cdot \lambda_{1}{ }^{2}-W_{c_{2}} \cdot \lambda_{2}{ }^{2}}{\lambda_{1}-\lambda_{2}} \times 10^{-8}
\end{aligned}
$$

$$
\begin{aligned}
D & =\frac{\frac{W_{c_{1}}}{f_{1}}-\frac{W_{c_{2}}}{f_{2}}}{f_{1}-f_{2}} \\
& =0.111 \ldots \frac{W_{c_{1}} \frac{\lambda_{1}^{2} \lambda_{2}-W_{c_{2}}}{\lambda_{2}-\dot{\lambda}_{1}} \dot{\lambda}_{2}^{2}}{\lambda_{1}} \times 10^{-16}
\end{aligned}
$$

where the wave length, $\lambda$, is again expressed in meters and $W_{c_{1}}$ and $W_{c_{2}}$ in watts.

Practical Hints On a Proper Differential Arrangement-A Discussion of Disturbances and Means of Overcoming Them

As can be seen from the introduction, the similarity and symmetrical arrangement of the two differential coils are a most important feature for the proper design of the transformer, because dissymmetry of the windings affects the inductance and resistance as well as the capacity phenomena of the primary coils with respect to the secondary. When these factors are not absolutely the same for each primary coil the phase of the radio frequency currents will be shifted unequally. All these conditions are complied with by the application of the so-called "ideal"-twisted wires. Approximately six turns in each primary coil with a diameter of about six inches $(15 \mathrm{~cm}$.) are recommended, 
in order to avert any unnecessarily large transformer losses. The same number of turns may be conveniently used for the secondary coil of the air transformer altho it is advisable to compute the number required for a particular zero current indicator.

Other disturbances present themselves because of the effect of the magnetic fields of the test sample and variometer in the secondary circuit of the transformer, and sometimes it is entirely impossible to cause the effects of the differential field to disappear completely. These influences are, however, overcome by arranging the test and comparison apparatus in such a way as to make their induction upon the transformer and secondary circuit a minimum. By choosing long leads on the one side (connecting to the transformer) the above-mentioned disturbances are practically eliminated.

Furthermore, it should be noted that inductive and capacity effects of the different parts of the arrangement with respect to each other are very pronounced within the range of radio frequency currents such as employed in radio telegraphy and telephony. Numerous investigations have shown that an accurate equalization of the differential system is practically impossible without extreme precautions. For this reason' a double cable (bifilar) enclosed in a grounded brass tube was used for all leads such as the mains which connect the apparatus to the radio frequency source and the leads connecting the zero current indicator with the differential transformer. One joint of the differential arrangement is also grounded (Figure 1) in order to cut down unnecessary leakage currents.

Further, it is interesting to study the case in which the resistance used for compensating the loss of the coil shows appreciable self induction and capacity effects. Consider the differential arrangement of Figure 9 as follows: The inserted series resistances, $r_{1}$ and $r_{2}$, each had a certain amount of self induction, $\Delta L_{1}$ and $\Delta L_{2}$, as well as a definite value of capacity, $\Delta C_{1}$ and $\Delta C_{2}$. Comparing the test sample with the variometer and these resistances, we obtain for the balance condition

hence

$$
\begin{aligned}
& r_{x}+r_{2}+j\left\{\omega\left[L_{x}+\Delta L_{2}\right]-\frac{1}{\omega \Delta C_{2}}\right\} \\
&=r_{s}+r_{1}+j\left\{\omega\left[L_{s}+\Delta L_{1}\right]-\frac{1}{\omega \Delta C_{1}}\right\}
\end{aligned}
$$

and the imaginary part gives the ratio

$$
\frac{L_{x}}{L_{s}}=1+\frac{1}{L_{s}}\left[\left(\Delta L_{1}-\Delta L_{2}\right)+\frac{1}{2}\left(\frac{\Delta C_{1}-\Delta C_{2}}{\Delta C_{1} \cdot \Delta C_{2}}\right)\right]
$$


which shows that the difference of self induction, $\Delta L_{1}-\Delta L_{2}$, has to be small in comparison with the effective self induction of the test sample. This condition is practically satisfied by the use of short length of manganin or constantin wire for the resistances $r_{1}$ and $r_{2}$. It is seen from equation (33) that the measurement of the difference of the resistance of test sample and variometer is not affected by any inductive or capacity effects of the

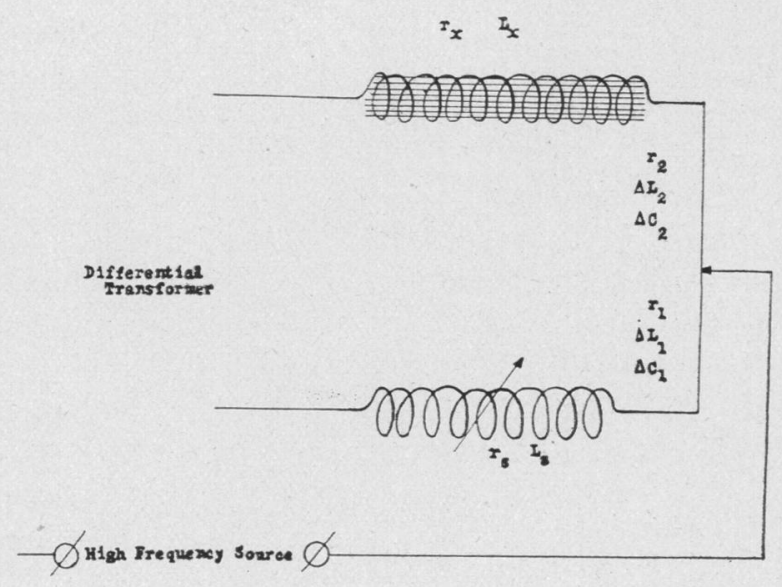

Figure 9

inserted balancing resistances, $r_{1}$ and $r_{2}$. This is an essential advantage of the differential method in comparison with the usually applied bridge arrangements, for the same inductive and capacity influences of the resistance introduce considerable errors at higher frequencies unless the bifilar bridge of Giebe is applied. The latter requires, however, an exact knowledge of the capacity and inductance of the leads, and is hardly available for measurements such as are met with in radio telegraphy. The influence of inductive effects of the resistances upon the loss adjustment, in case a bridge arrangement were used, may be seen fron Figure 10 and the following deductions. We then have as a general condition of balance

$$
\frac{r_{x}+j \omega L_{x}}{r_{s}+j \omega L_{s}}=\frac{r_{2}+j \omega \Delta L_{2}}{r_{1}+j \omega \Delta L_{1}}
$$

and separating the real and imaginary parts,

$$
r_{x} r_{1}-r_{s} \cdot r_{2}=\omega^{2}\left[L_{x} \Delta L_{1}-L_{s} \Delta L_{2}\right] .
$$


or

$$
\begin{gathered}
L_{x} r_{1}-L_{s} r_{2}=r_{s} \Delta L_{2}-r_{x} \Delta L_{1} \\
\frac{r_{x}}{r_{s}}=\frac{r_{2}}{r_{1}}+(2 \pi f)^{2}\left[\frac{L_{x} \Delta L_{1}-L_{s} \Delta L_{2}}{r_{1} r_{s}}\right] \\
\frac{L_{x}}{L_{s}}=\frac{r_{2}}{r_{1}}-\left[\frac{r_{x} \Delta L_{1}-r_{s} \Delta L_{2}}{L_{s} r_{1}}\right]
\end{gathered}
$$

Equation 38 shows clearly that the resistance adjustment is considerably affected at higher frequencies when inductive effects

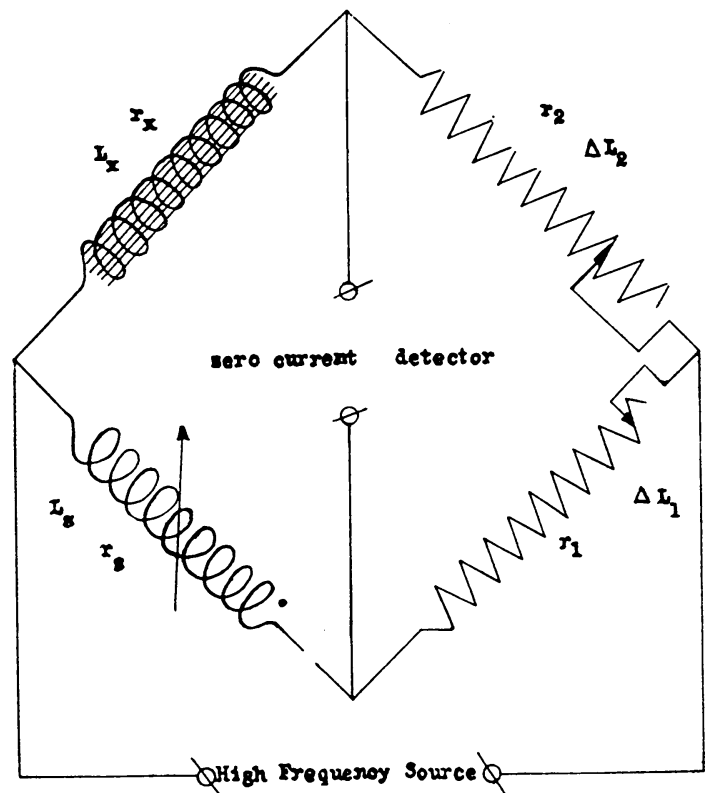

Figure 10

of $r_{1}$ and $r_{2}$ present themselves. Moreover, the bridge method is not advantageous since four branches act inductively on each other. Furthermore, three terms are to be varied for balance instead of only two and the protection of the detector circuit would cause grave difficulties.

The ordinary thermo-couple arrangement and barreter system are convenient expedients as zero current detectors, altho for very delicate readings the thermo-cross bridge is to be recommended.

Before concluding, it might be of interest to investigate somewhat the effects of the disturbing capacity with respect 
to the body of the observer and the like. For this purpose imagine that the test sample be balanced against the comparison standard. The zero current indicator will, however, only indicate a definite minimum at very high frequencies, even if resonance is established in the detector circuit by means of a condenser. Assume, further, that all the above precautions are employed and that the leads of the zero current detector are well protected against disturbances such as mentioned above. Yet the zero current detector will indicate a certain flux interlinked with the secondary circuit of the differential transformer. The cause of the disturbance can only be based upon capacity influences, which can be proved experimentally. For instance, when the observer touched different parts of the differential arrangement the telephone receiver (for this class of investigations, an oscillatory detector arrangement was employed as zero-current indicator) gave different sounds at the minimum. By putting the hand on one of the secondary terminals of the tranformer, the minimum was better. This phenomenon is due to charges and discharges causing a leakage current, flowing from the primary to the secondary turns of the differential transformer and such a stray current flowing from the turns of the telephone receiver thru the metal case and the hand of the observer to the ground. The primary coil, the secondary coil and the turns of the telephone receiver are regarded each as one pole of a condenser. This assumption can be made, as there will be not a strict mathematical treatment of this case; but this assumption is simply used for the proper interpretation of the cause of the above phenomena. Suppose, $V_{3}$, is the potential of the primary turns, $V_{2}$, that of the secondary coil, $V_{1}$, the potential of the turns of the telephone receiver, and the body of the observer has the potential $\mathrm{V}_{o}$. Assuming further that $\mathrm{C}_{32}$ is the capacity of the condenser formed by the primary and secondary coil of the differential transformer, and $\mathrm{C}_{10}$ the capacity of the condenser formed by the turns of the telephone receiver and the body of the observer. Then, from the primary to the secondary turns of the transformer a charging current, $\left[V_{3}-V_{2}\right] \omega C_{32}$, flows, of which a certain part, $\left[V_{1}-V_{o}\right] \omega C_{10}$, flows thru the hand to ground. Suppose we touch one of the secondary terminals of the transformer, that is, that the same is brought to the potential $V_{o}$. Consequently the second stray current disappears and the first one becomes, $\left(V_{3}-V_{o}\right) \omega C_{32}$. It might be believed, that the increase, $\left\{\left[V_{3}-V_{o}\right]-\left[V_{3}-V_{2}\right]\right\} \omega C_{32}$ would affect the telephone receiver more and would not dim- 
inish the sound. But if we bear in mind, that the body and therefore the potential $V_{o}$ ) is connected with one of the secondary terminals of the transformer, it is understood that practically most of the stray current will be led thru the observer to the ground. It would be a wrong expedient against these disturbances to ground one of the secondàry terminals, as that would only diminish the sensitiveness of the arrangement. Instead the writer used a tube of glass for handling the slide resistance and a cord for turning the coils of the standard variometer. By this means the current, $\left(V_{1}-V_{o}\right) \omega C_{10}$ could be made exceedingly small. (It is to be noted that for very precise measurements the telephone receiver is to be replaced by a galvanometer.) The first capacity current flowing from the primary to the secondary turns affected the telephone receiver much more. In order to overcome this disturbance the writer put a copper cylinder around the secondary turns. The cylinder consisted of enamelled copper wire. Along a longitudinal line the insulation was removed and all turns of the cylinder connected to ground. On an opposite longitudinal line, the protecting cylinder was cut in order that the damping action of the transformer might not be increased too much.

SUMMARY: The method described gives a ready means for determining the magnetizing force, the corresponding flux density, and permeability at any wave length whatever. In taking a series of readings for different ampereturns and at a definite wave length, we may obtain

(a) the magnetization curve,

(b) the permeability-ampere-turns curve.

Since the suggested arrangement applies to any practically available wave length we have a convenient means to compare the $B_{\max }$-values for very long wave lengths with the corresponding values determined at higher frequencies, and thus obtain a clear insight into the skin action of an iron core.

In a similar way the permeability-wave length curve may be found for a definite number of ampere turns. The method simultaneously determines either the total losses of the test sample or the losses due to direct current resistance, skin effect of the conductor, hysteresis and eddy currents in the iron core separately, and there can be obtained the

(a) watts/unit volume-wave length curve for a certain number of ampere turns,

(b) watts / unit volume-flux density curve at a constant wave length, and

(c) watts/unit volume-thickness of laminations curve for a constant wave length and a constant number of ampere turns; which enables the investigator to ascertain all the conditions which are necessary for determining the desired properties of any radio frequency apparatus containing a ferro-magnetic medium.

Moreover, by means of equations (14) and (15) one is able to experimentally investigate the dependance of the magnetizing force and permeability on the frequency. 


\section{LIST OF SYMBOLS USED}

$a$ Hysteresis exponent.

$\beta \quad$ Eddy current exponent.

$B_{\max }$ Maximum magnetic flux density, number of lines of magnetic induction per square centimeter.

$\left.\begin{array}{l}C_{10}, C_{32}, \\ \Delta C_{1}, \Delta C_{2},\end{array}\right\}$ Capacities in farads.

$d$ Thickness of iron lamination in centimeters.

i) Hysteretic angle of advance.

$E$ Effective induced E. M. F. of $N$ turns of the test sample.

$E_{a v} \quad$ Average induced voltage of a single turn.

\% Hysteresis constant.

$F$ Form factor.

$f$ Frequency.

$\Phi_{\max } \quad$ Maximum flux traversing the test coil.

$\Phi_{t} \quad$ Flux traversing the test coil at any time $t$.

$\phi$ Displacement of phase between terminal voltage and current of the test sample.

$H_{\max }$ Maximum resultant field intensity in gilberts per centimeter.

$I$ Current flowing to the differential system.

$I^{\prime \prime} / 2$ Effective current value of the component of the exciting current of the test sample containing the higher harmonics.

$I_{h} \quad$ Effective valte of hysteresis current.

$I_{m}$ Effective value of magnetizing component of exciting current.

$I^{\prime}{ }_{W} / 2$ Effective value of the energy component of the equivalent sine wave of the exciting current.

$I^{\prime}{ }_{W L} / 2$ Wattless component of the equivalent sine wave.

$i_{i} / 2$ Instantaneous value of the exciting current.

$i^{\prime} / 2$ Instantaneous value of the equivalent sine wave.

$i^{\prime \prime} / 2$ Instantaneous value of the component of the exciting current containing all higher harmonics.

$j=\sqrt{-1}$ The imaginary unit.

$\left.\begin{array}{l}K_{1}, K_{2}, k_{1}, \\ k_{2}, k_{3}, k_{4}, k_{3}\end{array}\right\}$ Constants. 
$\left.\begin{array}{c}\begin{array}{l}L_{s}, L_{s c}, L_{x}, L_{x c}, L_{2}, \\ \Delta L_{1}, \Delta L_{2}, \Delta L_{x}\end{array}\end{array}\right\} \begin{aligned} & \text { Coefficients of self induction in henrys. } \\ & \text { Length of magnetic path in centimeters. }\end{aligned}$

i. Wave length in meters.

$\mu \quad$ Magnetic permeability.

$N$ Number of turns of the test sample.

$r$ Series resistance in ohms.

$r_{x}{ }^{\prime} \quad$ Direct current resistance of test sample.

$r_{x}^{\prime \prime}$ Alternating current resistance of test sample.

$r_{x}$ Resistance of test sample under any condition.

$\Delta r_{c}$ Increase of the resistance of the test sample caused by core loss.

$\Delta r_{f}$ Increase of the resistance at the frequency $f$ caused by skin effect.

$S$ Cross sectional area of iron core in square centimeters.

$T$ Period of radio frequency current.

$\Gamma, V_{1}, V_{2}, V_{3}$ Terminal voltages.

$r$ Volume of iron core in cubic centimeters.

$W_{c}$ Total core loss in watts.

$W_{c_{1}}$ Total core loss in watts at a frequency $f_{1}$.

$W_{c_{2}}$ Total core loss in watts at a frequency $f_{2}$.

$W_{e} \quad$ Core loss due to eddy currents.

$W_{h}$ Core loss due to hysteresis.

(1) Angular velocity of radio frequency current.

$\xi \quad$ Eddy current constant.

$z$ Resistance operator of variometer-resistance combination.

$z_{x}$ Resistance operator of test sample when no iron is present.

$z_{x}{ }^{\prime} \quad$ Resistance operator of test sample when iron is present. 